Textures and Microstructures, Vol. 30, pp. 155-166 Reprints available directly from the publisher Photocopying permitted by license only
(C) 1998 OPA (Overseas Publishers Association) N.V.

Published by license under the Gordon and Breach Science Publishers imprint. Printed in India.

\title{
NEW POSSIBILITIES OF PHASE, TEXTURE, AND STRESS ANALYSIS USING ENERGY DISPERSIVE DETECTORS
}

\author{
K. MORAS * and R. ARNHOLD \\ Rich. Seifert \& Co Freiberger Präzisionsmechanik GmbH, Am St. Niclas \\ Schacht 13, D-09599 Freiberg, Germany
}

(Received 14 November 1996)

For angular dispersive and energy dispersive phase, texture, and stress analysis of compact samples (TiAl) and thin $\mathrm{TiN}$ layers, an electroluminescence detector and a $\mathrm{Si}(\mathrm{Li})$ semiconductor detector were applied. The parameters and application properties of the detectors are described. For angular dispersive measurements higher reflection intensities and better peak-to-background ratios can be attained, in comparison to conventional methods, by using multichannel pulse accumulation and mathematical spectrum evaluation.

In energy dispersive texture and stress measurements, reflection data from several lattice planes can be obtained simultaneously. In stress measurement, variations of lattice spacings in the order of $10^{-3}$ are reliably detectable. In principle, texture and stress information can be received from one measurement.

Keywords: Energy dispersive methods; Electroluminescence detector; $\mathrm{Si}(\mathrm{Li})$-detector; Texture analysis; Stress analysis; Mathematical spectrum evaluation

\section{INTRODUCTION}

For determining the texture of substances of low crystal symmetry or of thin surface layers, weak reflections must be included in general. This often leads to an insufficient accuracy and to long measuring times because of small intensities and poor peak-to-background ratios. Due to beam defocussing, in most cases the conditions cannot be improved

\footnotetext{
* Corresponding author. Fax: +49 3731781266.
} 
by using a crystal monochromator behind of the sample. In addition, the reflection efficiency of, e.g. a graphite monochromator is not better than $20-25 \%$. However, the application of detectors with high energy resolution and a sufficiently large entrance window allows a nearly lossless measurement of peak intensity at strongly reduced background. In previous investigations (Moras and Richter, 1992), we attained a three to five-fold intensity gain by using an electroluminescence detector instead of a secondary graphite monochromator for phase analysis of $\mu \mathrm{m}$-TiN layers on steel.

The aim of the present work is to investigate the principal possibilities of the application of detectors of different energy resolution (electroluminescence and semiconductor detectors) in the angular dispersive or energy dispersive mode for phase, texture, and stress analysis. At the same time, the advantages and limitations of single channel versus multichannel pulse accumulation including mathematical spectrum evaluation are analyzed.

The present results are of preliminary character, the investigations are not yet finished.

\section{EXPERIMENTAL SETUP}

For the new experiments, a SEIFERT XRD 3000/TS diffractometer in reflection geometry with a $\mathrm{Cu}$ long fine focus tube was applied. For the radiation detection we used:

(a) The completely new designed electroluminescence detector LUX from SEIFERT, coupled to the multichannel analyzer (MCA) card TRUMP 2K from EG\&G ORTEC.

(b) The Energy dispersive X-ray spectrometer with $200 \mathrm{~mm}^{2} \mathrm{Si}(\mathrm{Li})$ detector, 3,51 Dewar, signal processing unit EDR288 and MCA processor from RÖNTEC.

Table I shows the main physical parameters of the two detection systems.

In Fig. 1 the physical principles of LUX detector are shown. The detector chamber, filled with ultra-pure Xenon gas, is divided by an electrical grid into the absorption zone and the luminescence zone. The electrons, obtained by ionization of the Xenon atoms by absorbed 
TABLE I Parameters of energy dispersive detectors

\begin{tabular}{lcc}
\hline Parameter & $\begin{array}{c}\text { LUX } \\
(\text { SEIFERT) }\end{array}$ & $\begin{array}{c}\text { Si }(\text { Li })+\text { EDR288 } \\
(\text { RÖNTEC) }\end{array}$ \\
\hline Energy range & $3-25 \mathrm{keV}$ & $(0.5) 3-40 \mathrm{keV}$ \\
Entrance window- $\varnothing$ & $20 \mathrm{~mm}$ & $16 \mathrm{~mm}$ \\
Energetic resolution for & $\leq 560 \mathrm{eV}$ & $\leq 250 \mathrm{eV}$ \\
MnK $_{\alpha}$ (FWHM) & $(9.5 \%)$ & $(4.2 \%)$ \\
Max. pulse rate $_{\text {Efficiency for CuK }}$ & $50,000 \mathrm{cps}$ & $20,000 \mathrm{cps}$ \\
at 25 keV & $95 \%$ & $98 \%$ \\
at 40 keV & $25 \%$ & $90 \%$ \\
Working conditions & & $35 \%$ \\
\hline
\end{tabular}

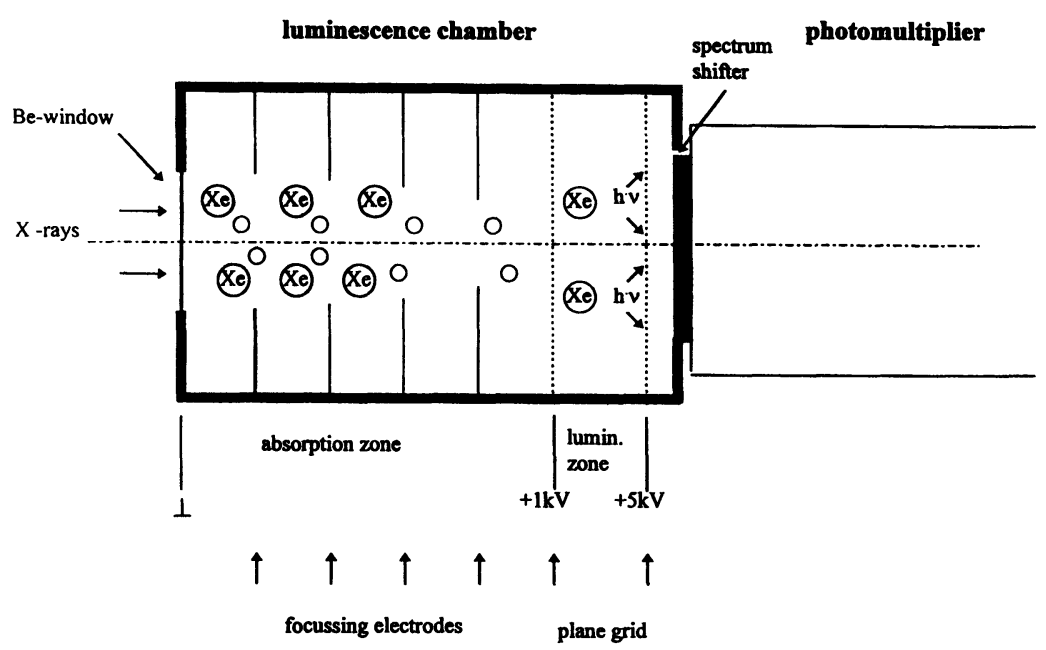

FIGURE 1 Illustration of the physical principle of the LUX detector.

$\mathrm{X}$-ray photons, drift in the relatively weak field into the luminescence zone. There the field strength is adjusted to such a value, that the Xe atoms are being excited to optical lumineseence, but that no impact ionization occurs. The gas amplification used in proportional counters is replaced here by a light amplification. The light photons are registered by the coupled photomultiplier (PMT). The relations are so, that the energy resolution is determined practically by the statistics of the primary ionization alone; the contributions of the light and PMTamplification are negligible. That is why the energetic resolution of the LUX detector is better by a factor 2 than that of a proportional counter, or by a factor 4 in comparison with a scintillation detector. 


\section{RESULTS AND DISCUSSION}

Some experiments on angular dispersive and energy dispersive diffractometry were carried out on TiN layers. Figure 2 shows the energy spectrum of a TiN layer on steel measured with a $\mathrm{Si}(\mathrm{Li})$ detector at $2 \vartheta=20^{\circ}$ (background position). On the left side the fluorescence peaks from $\mathrm{Ti}$ and $\mathrm{Fe}$ are seen, additionally the electronically generated zero peak for calibration purposes. The $\mathrm{Cu}$ peaks are from diffuse scattering of copper radiation (they are no Bragg peaks). On the right side there are Laue peaks caused by the reflection of white radiation at TiN lattice planes. These peaks shift to lower energies when $2 \vartheta$ increases.

In Fig. 3 the spectrum of a TiN(Fe) layer on a $\mathrm{Si}$ substrate is shown, measured with a LUX detector at low incidence angle and at the (220) reflection position $\left(2 \vartheta=62^{\circ}\right)$. The energy resolution is a bit worse here so that there is some overlapping between $\mathrm{Cu}$ and $\mathrm{Fe}$ peaks.

On the basis of these spectra the different measuring modes for diffractometry with energy dispersive detectors can be illustrated.

(a) Angular dispersive $(A D)$ diffractometry with a single channel analyzer ( $S C A$ ). The analyzer levels are to be set on the left and right side of the $\mathrm{CuK}_{\alpha}$ Peak, no peak profile evaluation is used. This simple method can be successfully applied if no peak overlapping

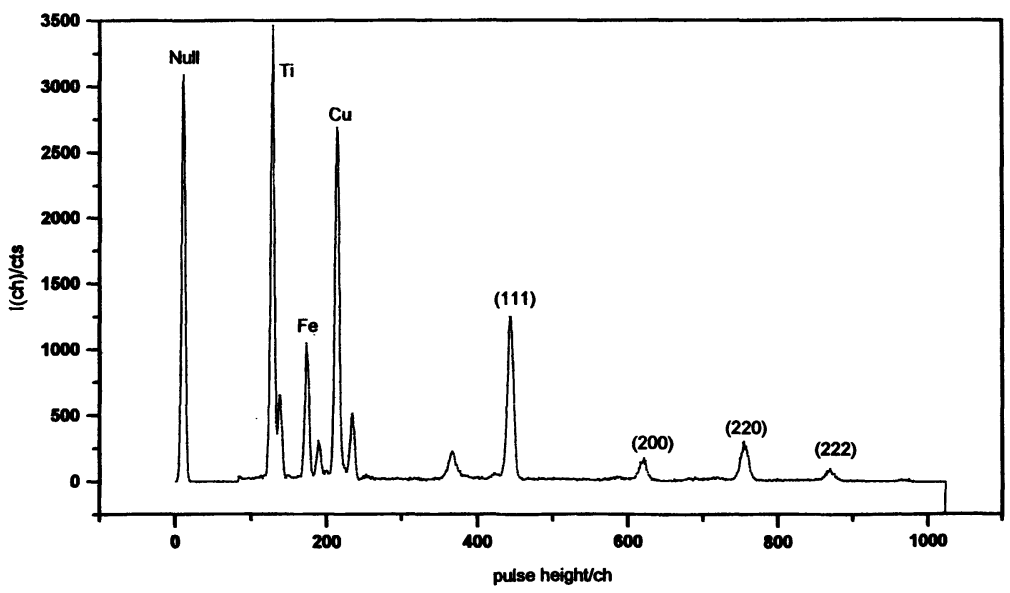

FIGURE 2 Energy spectrum of a TiN layer on steel, measured with $\mathrm{Si}(\mathrm{Li})$ detector $(240 \mathrm{eV}), \mathrm{Cu}$ tube $40 \mathrm{kV}, 20 \mathrm{~mA}, 2 \vartheta=20^{\circ}, t=200 \mathrm{~s}$. 


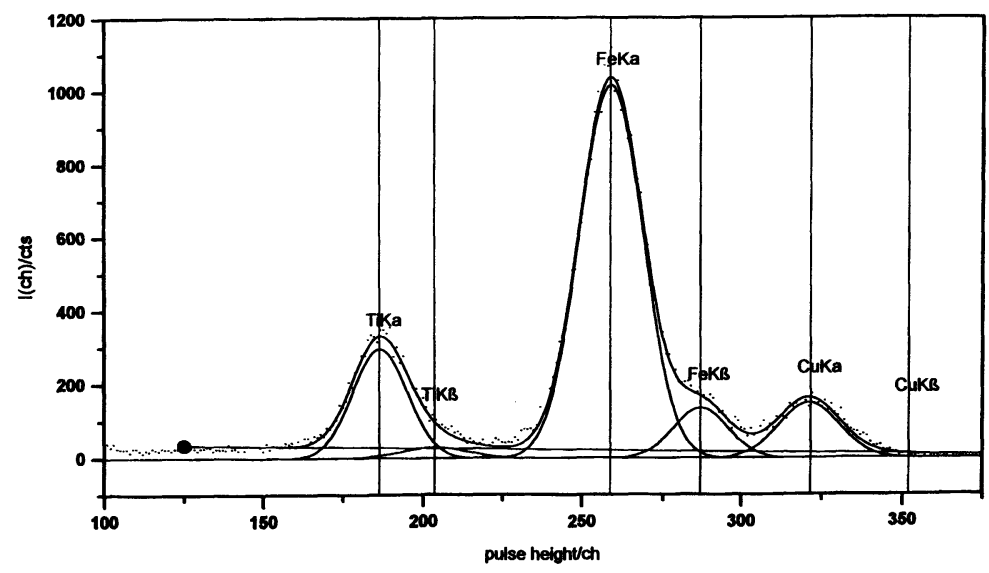

FIGURE 3 Energy spectrum of a $\mathrm{TiN}(\mathrm{Fe})$ layer on $\mathrm{Si}$, measured with a LUX detector at $\operatorname{TiN}(220)$ reflection position, $\mathrm{Cu}$ tube $40 \mathrm{kV}, 30 \mathrm{~mA}, 2 \vartheta=62^{\circ}, \omega=3.75^{\circ}$, $t=100 \mathrm{~s}$.

occurs, that means in many cases when using a semiconductor detector but only relatively seldom with the electroluminescence detector.

(b) Angular dispersive diffractometry with a multichannel analyzer $(M C A)$. In this mode, at each angular position the whole pulse height spectrum is being recorded and a certain range around the $\mathrm{CuK}_{\alpha}$ peak is evaluated by peak profile analysis in order to correct for peak overlapping and background. In the ideal case, this procedure results in almost $100 \%$ monochromatic $\mathrm{K}_{\alpha}$ intensity. Results using the LUX detector for this method are shown below.

(c) Energy dispersive diffractometry with an $M C A$. The energy spectrum is recorded at suitable angular positions and the Laue peaks are evaluated for peak position and integral intensity, if necessary, including overlap correction. The simultaneously measured Bragg and fluorescence peaks can be used for calibration purposes, the latter ones can give additional information on chemical composition or the thickness of metallic layers. For this mode, the $\mathrm{Si}(\mathrm{Li})$ detector is more suitable due to its higher energy resolution. The typically recordable energy range is from 10 to $40 \mathrm{keV}$ which corresponds to a d-value range, e.g., from 0.1 to $0.4 \mathrm{~nm}$ if a $2 \vartheta$ angle of $20^{\circ}$ is used. 
These measurement and evaluation methods can be used in connection with different diffractometer techniques, such as scans for phase analysis, for texture, or for stress analysis.

In a previous investigation (Bermig et al., 1993) texture analysis of a $\mathrm{Ti}-\mathrm{Al}$ sample containing the tetragonal $\gamma$ - and hexagonal $\alpha_{2}$-phases was carried out. For this analysis pole figures of some weak reflections had to be measured. Figure 4(a) shows the pulse height spectra of the sample measured with a scintillation (curve b) and an electroluminescence detector (curve a). It is evident that, due to the strong $\mathrm{Ti}$ fluorescence, with the scintillation counter no acceptable peak to background ratio can be achieved. On the other hand, using an electroluminescence detector in single channel mode, the $\mathrm{Cu}$ and Ti peaks could be completely separated and a well evaluable diagram was attained (Fig. 4(b)). This analysis could be carried out successfully only by using such an energy resolving detector.

Using the LUX detector, the conventional and the new detection modes were compared for $\mathrm{Ti}-\mathrm{Al}$ phase analysis. In Fig. 5 a part of the

(a)

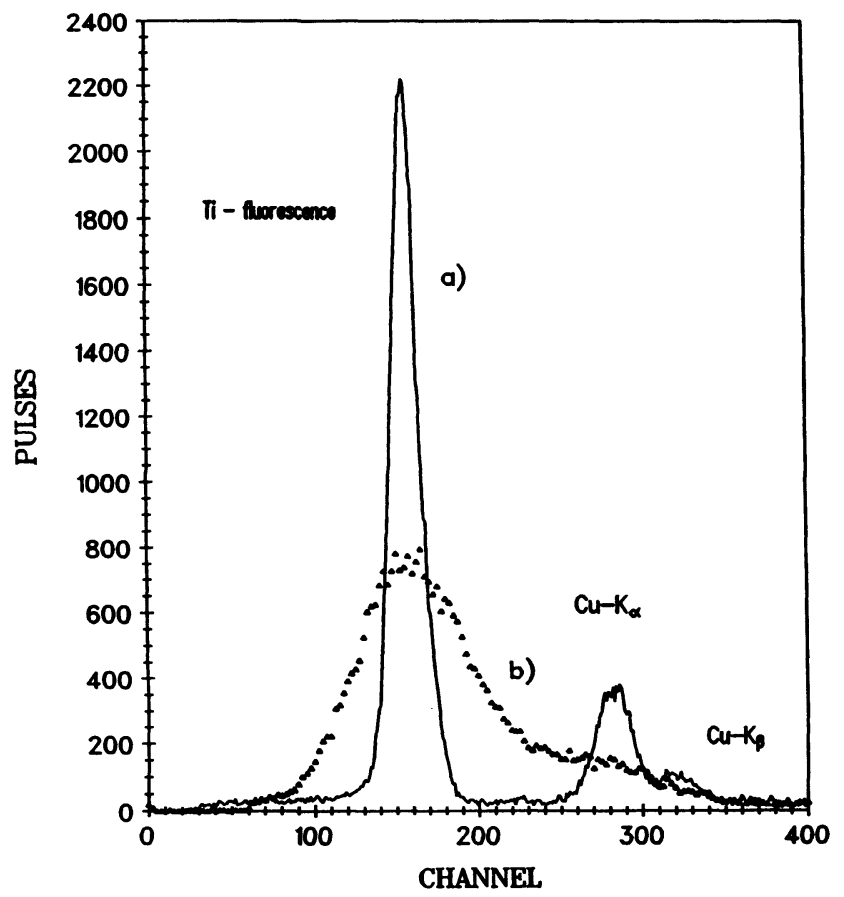


(b)

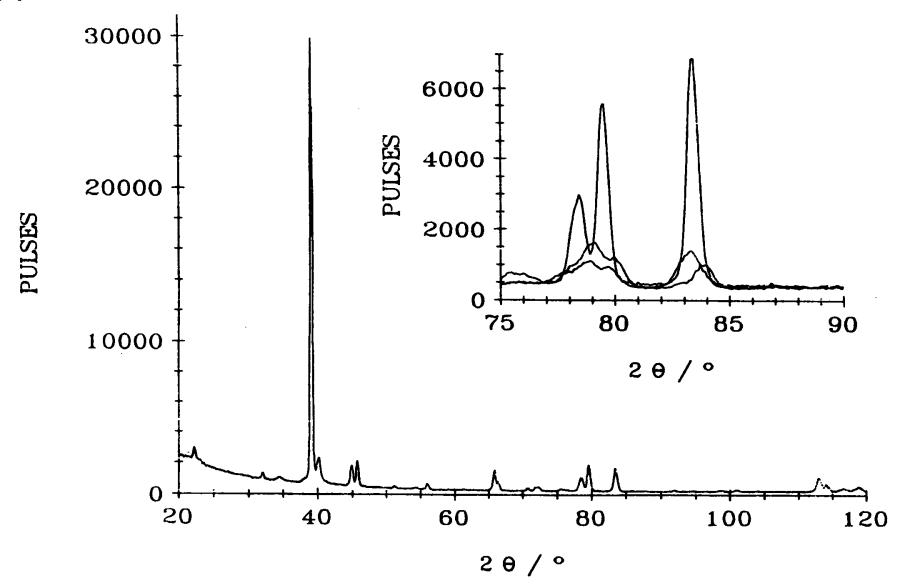

FIGURE 4 Diffraction analysis of a TiAl sample with different radiation detectors: (a) Energy spectrum of the sample with $\mathrm{Ti}$ and $\mathrm{Cu}$ fluorescence peaks measured by an electroluminescence (curve a) and a scintillation (curve b) detector. (b) Diffraction diagram showing reflections of the $\alpha_{2}$ and $\gamma$ phases. Detail: Some weak reflections at $\chi=0^{\circ}$ and $\chi=70^{\circ}$ which had to be measured for complete texture analysis.

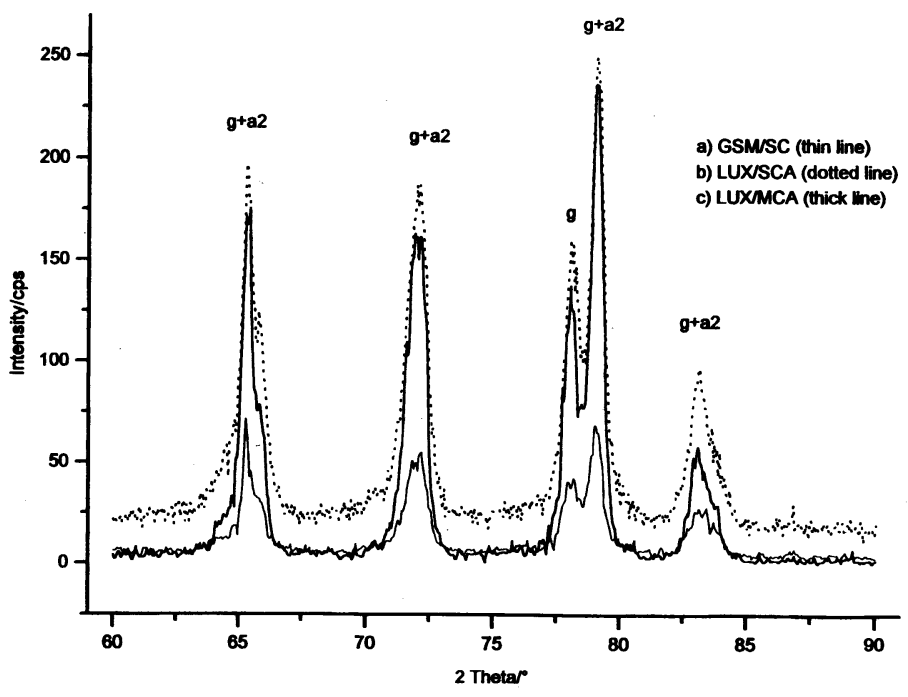

FIGURE 5 Diffraction diagram of TiAl (detail) with reflections of the $\alpha_{2}$ and $\gamma$ phases ( $a_{2}$ and $g$ in the diagram). Bragg-Brentano geometry. Detector: LUX in single channel (SCA) and multichannel (MCA) modes; scintillation counter with secondary graphite monochromator (GSM/SC). Cu tube $50 \mathrm{kV}, 30 \mathrm{~mA}, t=3 \mathrm{~s}$. 


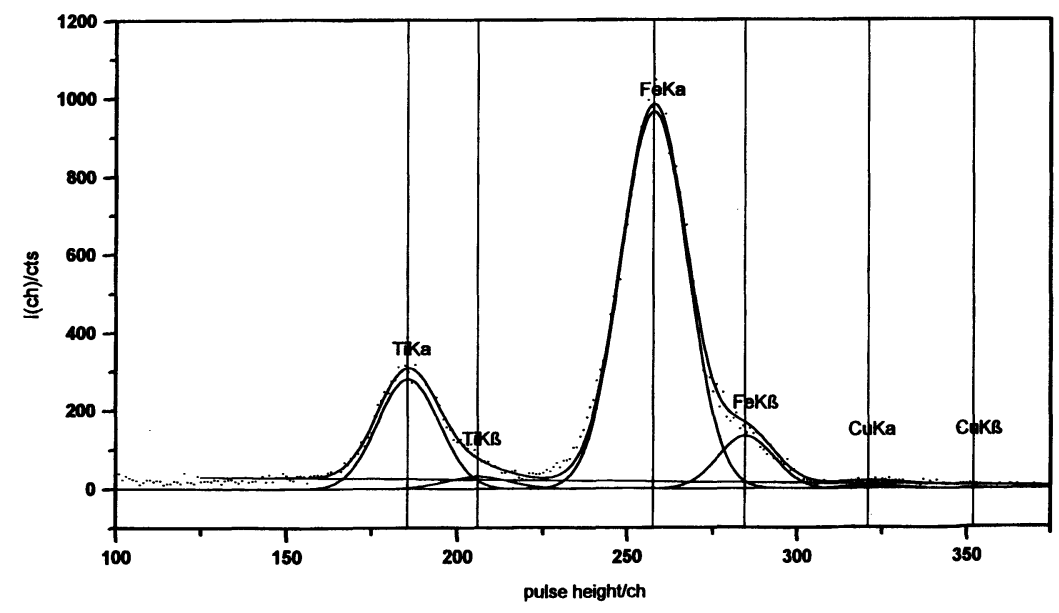

FIGURE 6 Energy spectrum of a TiN(Fe) layer on Si measured with a LUX detector at background position, $\mathrm{Cu}$ tube $40 \mathrm{kV}, 30 \mathrm{~mA}, 2 \vartheta=70^{\circ}, \omega=3.75^{\circ}, t=100 \mathrm{~s}$.

diffraction diagram is shown measured (a) with a secondary graphite monochromator + scintillation counter, (b) using a LUX detector with single channel analyzer, (c) with LUX detector + multichannel analyzer including mathematical curve fit. The use of a graphite monochromator succeeds in a very low background but leads to weak reflection intensities. With the LUX detector in SCA mode, high reflection intensities but also a higher background are attained. The application of the LUX detector in MCA mode results in high reflection intensities at a low background level almost as with graphite monochromator, corresponding a high peak-to-background ratio and good detection sensitivity.

It has to be remarked, that for the last measurement a relatively simple evaluation of the energy spectrum was made, i.e. a curve fit including only the $\mathrm{CuK}_{\alpha}$ and $\mathrm{K}_{\beta}$ peaks and a linear background function.

The evaluation algorithm in a much more complicated case of the energy spectrum was tested, carrying out an angular dispersive diffraction scan with MCA accumulation at the above-mentioned $\mathrm{TiN}(\mathrm{Fe})$ layer on Si substrate. See Figs. 3 and 6 for the energy spectrum, the first one at the (220) maximum, the second one at a background position, where the $\mathrm{CuK}_{\alpha}$ peak is missing, at constant (low) 
incidence angle $\omega$. There are six overlapping peaks. The Ti peaks should not really affect on the $\mathrm{CuK}_{\alpha}$ peak, but they were included here for testing. In some preliminary tests it was found that Gauss functions are suitable to fit the peaks, but that some narrow, physically founded, bounds and constraints for the peak parameters are necessary during the fitting procedure in order to ensure the convergence of the algorithm. Figure 7 shows a part of the diffraction diagram. The points of the lower curve represent the integral over the $\mathrm{CuK}_{\alpha}$ peak function corrected for background. It must be pointed out, that exactly the same algorithm, without parameter changes, was applied to all Bragg angles. That means an automatic fitting procedure for the whole diagram is possible.

For comparison, in the upper curve of Fig. 7, the measured values for SCA mode are shown, corresponding to a "window" from ch 300 to ch 344 (see Fig. 3). It is evident, that by the MCA mode, including spectrum evaluation, the background is reduced to $25 \%$ whereas the net intensity remains at approximately $\mathbf{9 0 \%}$. The peak-to-background ratio is improved by a factor of 2.5 . Compared to a measurement with a secondary graphite monochromator it can be estimated, that an improvement of the intensity-to-background ratio by a factor of 5-8 should be attainable. (Remark: Because it was only a test investigation,

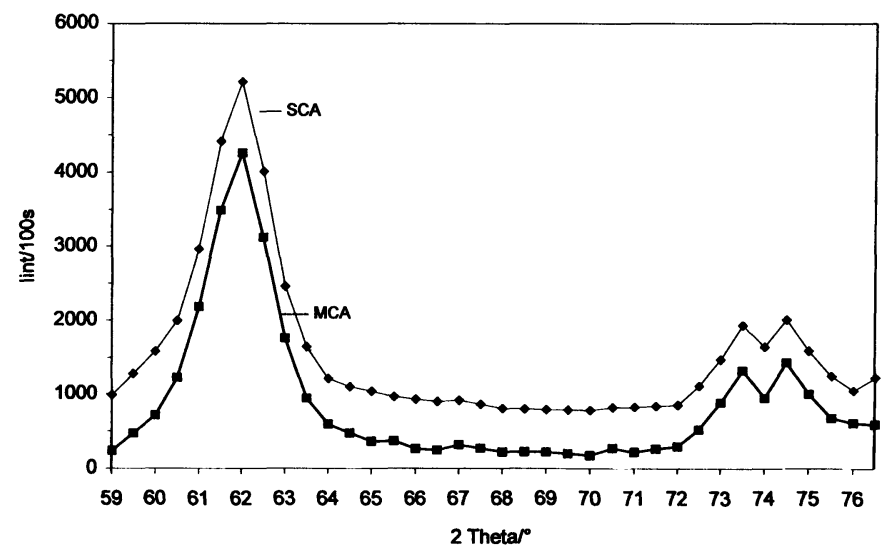

FIGURE 7 Diffraction diagram of a $\mathrm{TiN}(\mathrm{Fe})$ layer on $\mathrm{Si}$ (detail) measured with a LUX detector: (a) in single channel mode accumulated between ch $300-344\left(\mathrm{CuK}_{\alpha}\right.$ peak region); (b) in multichannel mode (MCA) with deconvolution of the $\mathrm{CuK}_{\alpha}$ peak from the overlapping $\mathrm{Ti}, \mathrm{Fe}, \mathrm{CuK}_{\beta}$ peaks and background (Cu tube $40 \mathrm{kV}, 30 \mathrm{~mA}$ ). 
the angular steps in Fig. 7 were chosen relatively large $\left(0.5^{\circ}\right)$, too large for good angular resolution.)

As an example for energy dispersive diffractometry using the $\mathrm{Si}(\mathrm{Li})$ detector we have investigated the possibilities and sensivity of texture and stress determination in a TiN layer on steel. For the energy spectrum of this sample look at Fig. 2 above. The Laue peaks of four TiN reflections are visible, additionally there two unidentified Laue peaks from the substrate (at ch 367 and ch 423). For the chosen $2 \vartheta$ angle $=20^{\circ}$, there is no overlapping with fluorescence or $\mathrm{CuK}_{\alpha} \mathrm{Bragg}$ peaks. The spectrum was accumulated for five tilting angles $\chi$ between $0^{\circ}$ and $50^{\circ}$ at $\varphi=0^{\circ}$, and the six Laue peaks were fitted by Gauss functions in a single procedure. An overlapping correction was necessary only for the TiN(111) peak with the peak at ch 423. In Fig. 8 the dependence of the integral intensity of the six peaks on the angle $\chi$ is shown, this corresponds, in principle, to sections across the pole figures for each peak at $\varphi=0^{\circ}$. It must be remarked here, that the measuring conditions here were not optimum for texture analysis (too large $\chi$ steps) because they were chosen for stress analysis (see below).

The measured spectra for the various $\chi$ angles were evaluated also concerning peak shift which is to be expected if the sample has

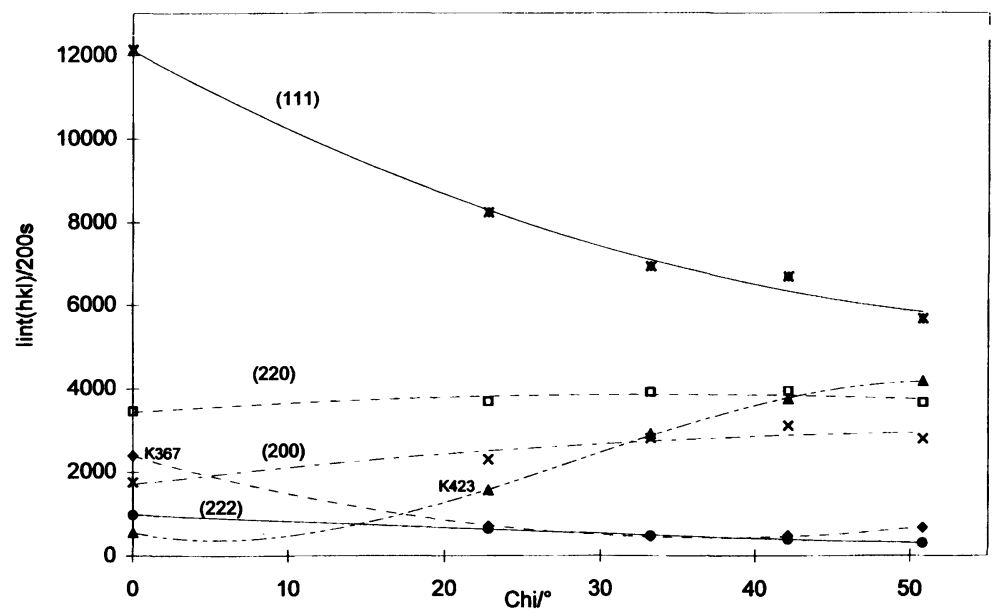

FIGURE 8 Energy dispersive simultaneous measurement of the integral intensity of 6 reflections of a textured and stressed TiN layer on steel in dependence on the tilting angle $\chi\left(\mathrm{Cu}\right.$ tube $40 \mathrm{kV}, 40 \mathrm{~mA}, 2 \vartheta=20^{\circ}, \mathrm{Si}(\mathrm{Li})$ detector $\left.16 \mathrm{~mm} \varnothing \mathrm{FWHM}=240 \mathrm{eV}\right)$. 
internal stress. In Fig. 9 the dependence of peak position and d-value on the $\chi$-angle is shown for the (220) reflection. The following conclusions can be drawn from the results.

(a) The observed peak shifts are small. This requires a high precision of the determination of the peak position, that means a sufficiently good counting statistics and an accurate peak approximation.

(b) Changes of the lattice constant $\mathrm{d}$ in the order of $10^{-3}$ are reliably detectable.

It should be noted, that the used $\mathrm{Si}(\mathrm{Li})$ detector with an energy resolution of $\leq 250 \mathrm{eV}$ was not optimal for stress investigations. With a detector of better resolution (e.g. $\leq 140 \mathrm{eV}$ ) a higher precision for $\mathrm{d}$ value determination should be attainable.

In summary, it must be emphasized, that from the measured and evaluated energy dispersive spectra, the texture and the stress information of the (in this example) six peaks could be obtained simultaneously. In practise, however, this requires a compromise concerning the measurement conditions (point or line focus of the tube; slits or collimators).

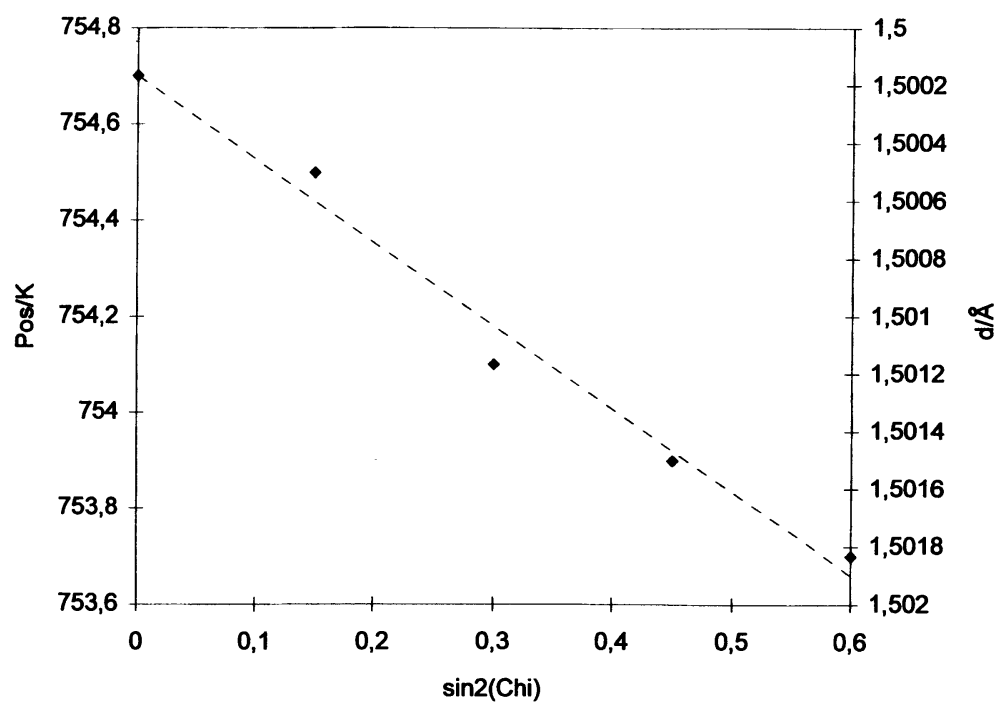

FIGURE 9 Energy dispersive measurement of the peak position and d-value of the (220)-lattice plane of a stressed TiN layer on steel in dependence on the tilting angle $\chi$ (Cu tube $40 \mathrm{kV}, 40 \mathrm{~mA}, 2 \vartheta=20^{\circ}, \mathrm{Si}(\mathrm{Li})$. detector $16 \mathrm{~mm} \varnothing \mathrm{FWHM}=240 \mathrm{eV}$ ). 


\section{CONCLUSIONS}

The investigations concerning employment of energy dispersive detectors in the X-ray diffraction phase, texture, and stress analysis lead to the following remarkable results.

- In the angular dispersive phase and texture analysis, by the application of energy dispersive detectors in connection with multichannel pulse accumulation and mathematical spectrum evaluation, substantially higher peak intensities and better peakto-background ratios can be attained in comparison to conventional methods.

- For angular dispersive analysis with an electroluminescence detector, especially with the newly developed type LUX, good results are obtained. For energy dispersive texture and stress analysis the $\mathrm{Si}(\mathrm{Li})$ semiconductor detector is more suitable due to its better energy resolution.

- In energy dispersive texture and stress analysis the parameters of several diffraction peaks can be recorded simultaneously. In stress measurement, variations of the lattice spacing in the order of $10^{-3}$ are reliably detectable. Making a compromise in optimum measuring conditions, texture and stress information can be obtained simultaneously from the same measurement.

\section{References}

Bermig, G., Tobisch, J., Richter, K. and Helming, K. (1993). New opportunities in X-ray texture analysis of two phase Ti-Aluminides by application of a proportional scintillation detector and the component method of ODF reproduction. Material Science Forum, Vol. 133-136 (1993) pp. 163-168.

Moras, K. and Richter, K. (1992). On the performance of the electroluminescence detector ELD65 in X-ray diffractometry. Symposium on X-ray Diffractometry in Materials Science, June 25-26, 1992, Freiberg/Sa. 